\title{
Risk for Coronavirus Diseases (COVID-19) among Cancer Patients from a Nursing Perspective
}

\author{
Nada Lukkahatai, PhD, MSN, RN, FAAN ${ }^{1 *}$, Aliki Argiropoulos, BSN, RN, OCN ${ }^{2}$ and Leorey N Saligan, \\ PhD, RN, CRNP, FAAN ${ }^{3}$
}

${ }^{1}$ Assistant Professor, School of Nursing, Johns Hopkins University, Baltimore, USA

${ }^{2}$ Nurse Clinician II, Blood and Marrow Transplant, Oncology Critical Care, Sidney Kimmel Comprehensive Cancer Center, The Johns Hopkins Hospital, USA

${ }^{3}$ Chief, Symptoms Biology Unit, National Institute of Nursing Research, Bethesda, USA

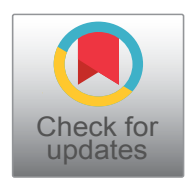

*Corresponding author: Dr. Nada Lukkahatai, PhD, MSN, RN, FAAN, Assistant Professor, School of Nursing, Johns Hopkins University, 525 N. Wolfe St. Baltimore, MD 21205, USA, Tel: 410-614-5297

\begin{abstract}
With this existing pandemic, it is critical to synthesize information that will be useful to frontline health care providers, particularly our nurses who are taking care of vulnerable populations. This commentary describes the common manifestations of COVID-19 infection, current management based on recent publications, hoping to equip nurses and other healthcare providers, especially those taking care of cancer patients, with some basic information about the signs and symptoms of COVID-19 infection.
\end{abstract}

\section{Keywords}

Coronavirus, COVID-19, Cancer

A viral outbreak, the novel coronavirus (COVID-19), has created an enormous clinical and public health crisis. The first known case of atypical pneumonia was reported on November 16, 2019, in China [1]. Within time, it has spread around the world. As of March 11, 2020, the World Health Organization declared it as a pandemic, as cases multiplied across 114 countries worldwide [2]. There have been over 182,000 cases worldwide, which is expected to gradually increase [3]. An army of nurses is in the frontlines to fight against this pandemic. Some nurses may not have enough information about the signs and symptoms related to this infection. This commentary describes the common manifestations of COVID-19 infection, current management based on recent publications, hoping to equip nurses and other healthcare providers, especially those taking care of cancer patients, with some basic information about the signs and symptoms of COVID-19 infection. Various health organizations have published a list of symptoms to be aware of, including fever, shortness of breath, and dry cough. While everyone is at risk for contracting COVID-19, there are serious concerns for patients with cancer, those receiving cancer therapy, and even those with a history of cancer. Evidence has shown that patients with cancer are at higher risk to develop serious events associated with COVID-19 infection [4]. Several recommendations have been put forward by several organizations for patients with cancer, based on past experiences with this virus. The hallmark symptoms reported by patients infected by COVID-19 include fever, shortness of breath, and dry cough [4-6]. Other articles also mentioned reports of chest tightness, diarrhea, and myalgia $[7,8]$. Chinese Center for Disease Control (CDC) divided clinical manifestations of COVID-19 into 3 categories include [1]:

1. Mild disease: Non-mild pneumonia, upper respiratory tract viral infection (mild fever, dry cough, muscle pain or malaise) without a sign of severe pneumonia (81\% of the cases)

2. Moderate disease: Fever, severe dyspnea, respira-

Citation: Lukkahatai N, Argiropoulos A, Saligan LN (2020) Risk for Coronavirus Diseases (COVID-19) among Cancer Patients from a Nursing Perspective. Int Arch Nurs Health Care 6:143. doi.org/10.23937/24695823/1510143

Accepted: April 07, 2020: Published: April 09, 2020

Copyright: (c) 2020 Lukkahatai N, et al. This is an open-access article distributed under the terms of the Creative Commons Attribution License, which permits unrestricted use, distribution, and reproduction in any medium, provided the original author and source are credited. 
tory distress, tachypnea, and hypoxia blood oxygen saturation $\left(\mathrm{SpO}_{2}\right)<90 \%$ and 3$)$ severe/critical disease ( $14 \%$ of the cases)

3. Severe/Critical disease: Respiratory failure, septic shock, and/or multiple organ dysfunction (MOD) ( $5 \%$ of the cases)

Studies described a cardinal sign associated with COVID-19 infection which includes an observable lung abnormality from computed tomography (CT) images before patients even reported any respiratory symptom $[7,9,10]$. A study reported that these lung changes were accidentally noticed from post-operative CT scans on two patients, following lobectomies for lung cancer [7]. By the time the two patients were experiencing respiratory symptoms (e.g., shortness of breath, chest tightness, dry cough, wheezing, and difficulty breathing), CT scans already showed an extensive geographic distribution of lung abnormalities (e.g., ground-glass opacity, reverse-halo consolidation). This supports the fact that there are physical signs in the early-phases of COVID-19, before experiencing specific symptoms.

A recent analysis of COVID-19 patients with cancer in China was conducted by Liang and colleagues [4]. From that analysis, it revealed that out of 1,590 COVID-19 cases, 18 of these patients had cancer or a history of cancer, which is roughly $1 \%$ of the total cases. This rate is higher overall than the Chinese general population. Compared to non-cancer COVID-19 patients, patients with cancer were older, with a median age of 63-years-old versus 48-years-old among the other non-cancer patients. In addition, $22 \%$ of the patients with cancer had a history of smoking, compared to $7 \%$ among non-cancer patients. This is an important factor, noting that smoking history has an increased susceptibility for COVID-19 infection [4]. Smoking is also the leading cause of COPD, which has been acknowledged as an independent risk factor for severe COVID-19 cases [11]. The most important discovery in the Liang and colleagues [4] study was that cancer patients had a $30 \%$ more risk of severe events compared to non-cancer patients, such as higher Intensive Care Unit admission, more cases who are mechanically ventilated, and higher mortality. Additionally, cancer patients' conditions deteriorated more swiftly to an average of 13 days compared to 43 days in non-cancer patients. Among cancer patients, those who received treatment within the past month had a higher risk ( $75 \%$ risk) of severe events than non-cancer patients (25\% risk) [4].

At present, in addition to the clinical manifestation, a standard definitive diagnosis of COVID-19 infection is the reverse-transcription polymerase chain reaction (RT-PCR) test or gene sequencing of the specimen collected by the oropharyngeal swab $[12,13]$. To date, there is no specific antiviral treatment recommended and no vaccine is available for COVID-19. In January 2020 , the WHO released guidelines addresses measures for categorizing patients by severity of the respiratory symptoms; early diagnosis and supportive therapy; and prevention and control strategies [5]. While scientific research is working on developing and testing coronavirus treatments and vaccines, WHO and other organizations urge the public to use preventive strategies. They issued the general recommendations to avoid public gatherings, frequent hand washing or the use portable hand sanitizer, and avoid contact with face and mouth after interacting with a possibly contaminated environment [5].

In response to these data, many medical organizations have instituted procedures to reduce the risk of cancer patients during the COVID-19 pandemic. It is important to note that those who are currently undergoing cancer treatment, or even those with a history of cancer, are more likely immune compromised and are more susceptible to infections. One example is at the Mount Sinai Health System, located in New York City, where cancer patients are require to have routine or non-urgent appointments to reschedule to a later date. Prior to arrival for an active treatment, patients with cancer are screened 24-48 hours by telephone before their scheduled appointments. Upon arrival in the facility, these patients undergo a full risk assessment in an isolation room. They are also provided one-page handouts with information about the flu and COVID-19. Patients with cancer also have access to updated hospital precautions related to this infection with a link to the Center for Disease Control website [14]. Another group recommended online medical counseling for any cancer patient with questions and concerns [15]. Mental health should also be seriously addressed for cancer patients. For cancer patients with COVID-19 infection, they are advised to postpone adjuvant chemotherapy or elective surgeries especially for those with stable cancers, protective personal equipment was provided, and more intensive surveillance and/or treatment were required [4]. There are limited studies on COVID-19 infections among cancer patients [11]. Most of these articles are case reports involving 2-18 cases, often not generalizable to the general cancer population. Since this COVID-19 pandemic is on-going and ever-changing, we expect more articles and research to advance our understanding of the short-term and long-term consequences of COVID-19 infection, especially among the most vulnerable, such as patients with cancer. It's imperative to continue to synthesize available information to assist nurses and other health providers as we continue to address and curve this common, existential threat to humanity.

\section{Sources of Support}

None.

\section{Authors' Contribution}

In preparation of this manuscript, all authors contribute equality in writing, reviewing and finalizing the 
manuscript. All authors had read and approved this manuscript. There is no conflict $s$ in interest with any of the authors.

\section{References}

1. Wu Z, McGoogan JM (2020) Characteristics of and important lessons from the coronavirus disease 2019 (COVID-19) outbreak in China: Summary of a report of 72314 cases from the Chinese Center for Disease Control and Prevention. JAMA.

2. World Health Organization (2020) WHO director-general's opening remarks at the media briefing on covid-19-11 March 2020.

3. Johns Hopkins University and Medicine (2020) Coronavirus COVID-19 Global Cases by the Center for Systems Science and Engineering (CSSE) at Johns Hopkins University (JHU).

4. Liang W, Guan W, Chen R, Wang W, Li J, et al. (2020) Cancer patients in SARS-CoV-2 infection: A nationwide analysis in China. Lancet Oncol 21: 335-337.

5. Cascella M, Rajnik M, Cuomo A, Dulebohn SC, Di Napoli $R$ (2020) Features, evaluation and treatment Coronavirus (COVID-19). StatPearls, Treasure Island (FL), USA.

6. Lake MA (2020) What we know so far: COVID-19 current clinical knowledge and research. Clin Med (Lond) 20: 124127.
7. Tian S, Hu W, Niu L, Liu H, Xu H, et al. (2020) Pulmonary pathology of early-phase 2019 novel coronavirus (COVID-19) pneumonia in two patients with lung cancer. J Thorac Oncol.

8. Zhang H, Huang $Y$, Xie $C$ (2020) The treatment and outcome of a lung cancer patient infected with SARS-CoV-2. J Thorac Oncol.

9. Ai T, Yang Z, Hou H, Zhan C, Chen C, et al. (2020) Correlation of Chest CT and RT-PCR testing in coronavirus disease 2019 (COVID-19) in China: A report of 1014 cases. Radiology.

10. Bai HX, Hsieh B, Xiong Z, Halsey K, Choi JW, et al. (2020) Performance of radiologists in differentiating COVID-19 from viral pneumonia on chest $\mathrm{CT}$. Radiology.

11. Xia Y, Jin R, Zhao J, Li W, Shen H (2020) Risk of COVID-19 for patients with cancer. Lancet Oncol 21: e180.

12. Yang W, Cao Q, Qin L, Wang X, Cheng Z, et al. (2020) Clinical characteristics and imaging manifestations of the 2019 novel coronavirus disease (COVID-19): A multi-center study in Wenzhou city, Zhejiang, China. J Infect 80: 388393.

13. Yang W, Yan F (2020) Patients with RT-PCR confirmed COVID-19 and normal Chest CT. Radiology.

14. Jenkins K (2020) Covid-19: Older patients with cancer especially vulnerable. Medscape.

15. Wang H, Zhang L (2020) Risk of COVID-19 for patients with cancer. The Lancet Oncology 21: e181. 